J. Ito

Nagoya Math. J.

Vol. 37 (1970), 137-144

\title{
ON REDUCIBILITY OF PROVABILITY IN THE PRIMITIVE LOGIG [LO]
}

\author{
JIRO ITO
}

Dedicated to Professor Katuzi Ono on his 60th birthday

\section{Introduction.}

In the present paper, we would like to show a theorem concerning with reducibility of provability in the primitive logic. This theorem seems to suggest a procedure to find the proof-note of a given proposition which is provable in the primitive logic.

The formulations of the primitive logic $\boldsymbol{L O}$ and [LO] have been introduced in Ono [1], [2], [3], and [5]. The primitive logic is the logic having only two logical constants IMPLICATION $\rightarrow$ and UNIVERSAL QUANTIFICATION ( ), and has an interesting property that any logic belonging to intuitionistic series or to classical series can be faithfully interpreted in it (Ono [3], [4]).

In the proof-note of the logic [LO ], there are some propositions enclosed in pairs of brackets. Any proposition in proof-notes of [LO ] is said to be CLAD or BARE according as it is enclosed or is not enclosed in a pair of brackets. The followings are the inference rules of the logic [LO ]:

[F]: The step $\mathfrak{A}$ can be deduced from the step [थ].

[I]: The step []] can be deduced from the steps $\mathfrak{A}$ and $[\mathfrak{A} \rightarrow \mathfrak{B}]$.

$\left[\boldsymbol{I}^{*}\right]:$ The step $\mathfrak{A} \rightarrow \mathfrak{B}$ can be deduced from the fact that $\mathfrak{B}$ is deducible from [?].

[U]: The step $[\mathfrak{U}(t)]$ can be deduced from the step $[(x) \mathfrak{U}(x)]$ as far as $\mathfrak{A}(u)$ contains no free variable $x$ at all.

$\left[\boldsymbol{U}^{*}\right]$ : The step $(x) \mathfrak{H}(x)$ can be deduced from the fact that $\mathfrak{A}(t)$ is deducible for any variable $t$ whatever, i.e. from the fact that the step $\mathfrak{A}(t)$ is deducible from the step $\forall t:$.

Received March 31, 1969 
Any proof-note is understood as a practical discription of steps of reasonings. Every step is introduced and denoted by its introductory indexword. Any index-word is a sequence of letters, such as $\boldsymbol{A}, \boldsymbol{b}, \boldsymbol{c}, \cdots$ assuming the usual alphabetical order between them, including the null sequence. We denote a sequence of letters by an underlined single letter such as $\underline{p}$ or $\underline{t}$, and especially null sequence by $\emptyset$.

Here, we must further refer to [5] on some technical terms such as ORDER (Natural, Fundamental and Basis) of steps, REFERENCE STEP and ASSUMPTION STEP. In [5], K. Ono showed a characteristic feature of the logic $[\boldsymbol{L O}]$ by proving the theorem:

Any proposition occurring in a wasteless proof-note of a proposition $\mathfrak{P}$ is a subformula of the proposition $\mathfrak{\$}$.

In this paper, we shall show that this theorem may be described more precisely from a view-point on reducibility of provability.

In section 1, we shall introduce some expressions on $\boldsymbol{L} \boldsymbol{O}$-formulas such as REGULAR PARSING-FORM, $\tau$-KERNEL and $\sigma$-CONSTRUCTION with their PARSING-FACTORS and KERNEL.

In section 2, we shall prove the main theorem on reducibility of provability such as the theorem:

The provability of a given proposition $\mathfrak{A}$ is reducible to the fact that there are finite sequences of factors $\{\boldsymbol{\mu}\}$ and $\{\boldsymbol{\nu}\}$ which satisfy the following conditions; 1$)$ the $\tau$-kernel of $\mathfrak{A}$ by factors $\{\boldsymbol{\mu}\}$ is deducible from the assumptions $\{\boldsymbol{\mu}\}, 2)\{\boldsymbol{\mu}\}$ contains a $\sigma$-construction by factors $\{\boldsymbol{\nu}\}$ with that $\tau$-kernel of $\mathfrak{A}$ by factors $\{\boldsymbol{\mu}\}$ as its kernel, and 3) any formula of $\{\boldsymbol{\nu}\}$ is deducible from the assumptions $\{\boldsymbol{\mu}\}$.

\section{Parsing expression.}

Any formula which has no logical constants other than implication and universal quantification is called an LO-formula.

Firstly, we would like to introduce the parsing expressions.

(1. 1) Regular parsing-form

We introduce a PARSING-FORM with its FACTOR and KERNEL as follows;

An expression $\langle\lambda\rangle \mathfrak{A}$ which is called a parsing-form by a $\pi$-factor $\lambda$ with kernel $\mathfrak{A}$, is defined by

1) if $\lambda$ is a formula $\mathfrak{E},\langle\mathfrak{C}\rangle \mathfrak{A} \equiv 1) \mathfrak{C} \rightarrow \mathfrak{A}:$

1) $P \equiv Q$ means " $P$ is defined by $Q$ ". 
2) if $\lambda$ is a variable $x,\langle x\rangle \mathfrak{A} \equiv(x) \mathfrak{U}(x)$ :

3) otherwise, $\langle\lambda\rangle \mathfrak{A}$ is undefined.

Especially, if $\lambda$ is empty or denoted by $\lambda_{0},\left\langle>\mathfrak{A}\right.$ or $\left\langle\lambda_{0}\right\rangle \mathfrak{A} \equiv \mathfrak{A}$ is called a parsing-form by the empty-factor or $\lambda_{0}$ with kernel $\mathfrak{A}$.

For any $i(i \geqq 1)$, a parsing-form with kernel $\mathfrak{A}$ by $\pi$-factors $\left\{\lambda_{0}, \cdots, \lambda_{i}\right\}$, $\left\langle\lambda_{0}, \lambda_{1}, \cdots \cdot, \lambda_{i}\right\rangle \mathfrak{A} \equiv\left\langle\lambda_{i}\right\rangle\left(\left\langle\lambda_{0}, \cdots, \lambda_{i-1}\right\rangle \mathfrak{A}\right)$ recursively, where $\lambda_{0}$ is empty and each of $\lambda_{i}(i \geqq 1)$ is a formula or a variable, respectively. In the case that $\lambda_{i}$ is a formula each of $\lambda_{i}$ is called a $\pi$-formula, otherwise a $\pi$ variable.

From the definition of parsing-forms we have the following lemmas.

Lemma 1. Any parsing-form which has an LO-formula as its kernel is also an LO-formula.

Lemma 2. Any LO-formula is expressible in a parsing-form by $\pi$-factors.

Accordingly, corresponding to any $L O$-formula $\mathfrak{P}$, there is one and only one parsing-form which has an elementary formula as its kernel. This expression is called a regular parsing-form of $\mathfrak{P}$.

(1. 2) $\tau$-Kernel.

Let $\mathfrak{A}$ be a formula whose regular parsing-form is $\left\langle\lambda_{0}, \cdots, \lambda_{n}\right\rangle E$. The expression $\tau\langle\mu\rangle \mathfrak{A}$ which is called a $\tau$-kernel of $\mathfrak{A}$ by a $\tau$-factor $\mu$, is defined as follows:

1) If $\mu$ is a formula $\mathfrak{E}$, and $\lambda_{n}$ is $\mathfrak{E}, \tau\langle\mathfrak{E}\rangle(\langle\mathfrak{C}\rangle \mathfrak{B}) \equiv \mathfrak{B}$,

2) If $\mu$ is a free variable $t$ and $\lambda_{n}$ is a variable $x, \tau\langle t\rangle(\langle x\rangle \mathfrak{B}(x)) \equiv \mathfrak{B}(t)$,

3) otherwise, $\tau\langle\mu\rangle \mathfrak{A}$ is undefined,

where $\mathfrak{B}$ or $\mathfrak{B}(x)$ denotes $\left\langle\lambda_{0}, \cdots, \lambda_{n-1}\right\rangle E$. The $\tau$-factor $\mu$ is called a $\tau$-formula or a $\tau$-variable according as $\mu$ is a formula or a variable, respectively.

Especially, if $\mu$ is empty or denoted by $\mu_{0}, \tau\left\langle>\mathfrak{A}\right.$ or $\tau\left\langle\mu_{0}\right\rangle \mathfrak{A} \equiv \mathfrak{A}$, is called a $\tau$-kernel of $\mathfrak{U}$ by the empty-factor or $\mu_{0}$.

For any $i(i \geqq 1)$, a $\tau$-kernel of $\mathfrak{U}$ by $\tau$-factors $\left\{\mu_{0}, \cdots, \mu_{i}\right\}$

$$
\tau\left\langle\mu_{0}, \mu_{1}, \cdots, \mu_{i}\right\rangle \mathfrak{A} \equiv \tau\left\langle\mu_{i}\right\rangle\left(\tau\left\langle\mu_{0}, \cdots, \mu_{i-1}\right\rangle \mathfrak{A}\right)
$$

recursively, where $\mu_{0}$ is empty and each of $\mu_{j}(i \geqq j \geqq 1)$ is a $\tau$-formula or a $\tau$-variable according as $\lambda_{n-j-1}$ is a formula or a variable, respectively. 
(1. 3) $\sigma$-construction.

An expression $\sigma\langle\nu\rangle \mathfrak{A}$ which is called a $\sigma$-construction with kernel $\mathfrak{A}$ by a $\sigma$-factor $\nu$, is defined as follows;

1) If $\nu$ is a formula $\mathfrak{C}, \sigma\langle\mathfrak{C}\rangle \mathfrak{A} \equiv\langle\mathfrak{C}\rangle \mathfrak{A}$.

2) If $\nu$ is a variable $t, \sigma\langle t\rangle \mathfrak{A}(t) \equiv\langle x\rangle \mathfrak{A}(x)$

where $\mathfrak{A}(x)$ denotes the derived formula from $\mathfrak{A}(t)$ replacing $t$ in its position by $x$ which has no occurrence in $\mathfrak{A}(t)$. The $\sigma$-factor $\nu$ is called $\sigma$ formula or $\sigma$-variable according as $\nu$ is a formula or a variable, respectively.

Especially, if $\nu$ is empty or denoted by $\nu_{0}, \sigma<>\mathfrak{A}$ or $\sigma\left\langle\nu_{0}\right\rangle \mathfrak{A} \equiv \mathfrak{A}$ is called a $\sigma$-construction with kernel $\mathfrak{A}$ by the empty-factor or $\nu_{0}$. For any $i(i \geqq 1)$, a $\sigma$-construction with kernel $\mathfrak{A}$ by $\sigma$-factors $\left\{\nu_{0}, \cdots, \nu_{i}\right\}$,

$$
\sigma\left\langle\nu_{0}, \nu_{1}, \cdots, \nu_{i}\right\rangle \mathfrak{U} \equiv \sigma\left\langle\nu_{i}\right\rangle\left\langle\sigma\left\langle\nu_{0}, \cdots, \nu_{i-1}\right\rangle \mathfrak{U}\right)
$$

recursively, where $\nu_{0}$ is empty and each of $\nu_{j}(j \geqq 1)$ is a $\sigma$-formula or a $\sigma$ variable.

(1. 4) Modulation of inference rules.

Using our expressions the inference rules of the logic $[\boldsymbol{L O}]$ are modulated as follows;

[F]: The step $\mathfrak{A}$ can be deduced from the step [थ].

[I]: The step [ỉ] can be deduced from the steps $\mathfrak{A}$ and $[\sigma<\mathfrak{X}\rangle \mathfrak{B}]$.

$\left[\boldsymbol{I}^{*}\right]$ : The step $\langle\mathfrak{A}\rangle \mathfrak{B}$ can be deduced from the fact that $\tau\langle\mathfrak{X}\rangle(\langle\mathfrak{A}\rangle \mathfrak{B})$ is deducible from [थ].

$[\boldsymbol{U}]:$ The step $[\mathfrak{A}(t)]$ can be deduced from the step $[\sigma\langle t\rangle \mathfrak{A}(t)]$.

$\left[\boldsymbol{U}^{*}\right]$ : The step $\langle x\rangle \mathfrak{A}(x)$ can be deduced from the fact that $\tau\langle t\rangle(\langle x\rangle \mathfrak{A}(x))$ is deducible for any variable $t$ whatever, i.e., from the fact that the step $\tau\langle t\rangle(\langle x\rangle \mathfrak{H}(x))$ is deducible from the step $\left.[t] .{ }^{2}\right)$

Let $\Gamma$ be an ordered set of clad formulas or variables which belong to assumption steps of a step $\underline{s}$, arranged its index-word in the fundamental

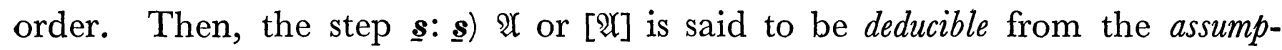
tion $\Gamma$. We would like to denote this by $\Gamma \vdash \mathfrak{A}$ or $\Gamma \vdash[\mathfrak{A}]$, respectively. Especially, if $\underline{s}$ is $\boldsymbol{\emptyset}$, the formula of $\underline{s}$ is bare and $\Gamma$ is empty. We denote this by $\vdash \mathfrak{A}$.

2) We will use clad variable $[t]$ instead of $\forall t$ :, because in a proof-note, denominating quantifier $\forall t$ : as well as clad formula [q] has an assumptional character. 
Any proposition $\mathfrak{B}$ is said to be provable in $[\boldsymbol{L O}]$ if and only if the step $\mathfrak{F}$ is deducible in $[\boldsymbol{L} \boldsymbol{O}]$.

\section{Reducibility of provability.}

\section{(2. 1) Lemmas.}

Now, we are going to prove the following lemmas preparatory to the main theorem.

Lemma 3. $\vdash\langle\lambda\rangle \mathfrak{A}$ if and only if $[\mu] \vdash \tau\langle\mu\rangle(\langle\lambda\rangle \mathfrak{A})$, where $\mu$ is a $\tau$-variable which has no occurrence in $\langle\lambda\rangle \mathfrak{A}$ or a $\tau$-formula according as $\lambda$ is a variable or $a$ formula, respectively.

Proof. Firstly we assume $\vdash\langle\lambda\rangle \mathfrak{A}$. In this proof-note, the assumption step of the step $\emptyset$ is empty, therefore the inference rule for the step $\emptyset$ is $\left[\boldsymbol{U}^{*}\right]$ or $\left[\boldsymbol{I}^{*}\right]$. Accordingly, there are steps $\left.\boldsymbol{A}\right)[\mu]$ and $\left.\boldsymbol{\epsilon}\right) \tau\langle\mu\rangle(\langle\lambda\rangle \mathfrak{A})$ in this proof-note, where $\mu$ is a $\tau$-variable which has no occurrence in $\langle\lambda\rangle \mathfrak{A}$ or a $\tau$-formula according as $\lambda$ is a variable or a formula. Thus we have $[\mu] \vdash$ $\tau\langle\mu\rangle(\langle\lambda\rangle \mathfrak{A})$. Conversely, we assume $[\mu] \vdash \tau\langle\mu\rangle(\langle\lambda\rangle \mathfrak{U})$ with the $\mu$-condition. Adding to this proof-note the step $\emptyset)\langle\lambda\rangle \mathfrak{A}$, we have $\vdash\langle\lambda\rangle \mathfrak{A}$ by $\left[\boldsymbol{U}^{*}\right]$ or $\left[\boldsymbol{I}^{*}\right]$ according as $\lambda$ is a variable or a formula, respectively.

In the following lemmas, let $\left\langle\lambda_{0}, \cdots, \lambda_{n}\right\rangle E$ be the regular parsingform of $\mathfrak{A}$.

Lemma 4. For any $k(n \geqq k \geqq 1)$, if $\left[\mu_{1}\right], \cdots,\left[\mu_{k}\right] \vdash \tau\left\langle\mu_{0}, \cdots, \mu_{k}\right\rangle \mathfrak{A}$, then $\vdash \mathfrak{A}$, where the $\mu$-condition: $\mu_{i}(k \geqq i \geqq 1)$ is a $\tau$-variable which has no occurrence in any $\tau\left\langle\mu_{0}, \cdots, \mu_{j-1}\right\rangle \mathfrak{A}(i \geqq j \geqq 1)$ or a $\tau$-formula according as $\lambda_{n-i+1} i$ r a variable or a formula, respectively, holds.

Proof. For $k=1$, the case is the last part of lemma 3.

Now, we assume this lemma for any number less than $k$, and prove it for $k$ by induction. In this proof-note, there are the step $\underline{s}) \tau\left\langle\mu_{0}, \cdots\right.$, $\left.\mu_{k}\right\rangle \mathfrak{A}$ and its assumption step $\left.\underline{\boldsymbol{u}} \boldsymbol{A}\right)\left[\mu_{k}\right]$ with the $\mu$-condition. Therefore, adding the step $\underline{\boldsymbol{u}}) \tau\left\langle\mu_{0}, \cdots, \mu_{k-1}\right\rangle \mathfrak{A}$ to this proof-note, we have $\left[\mu_{1}\right], \cdots$, $\left[\mu_{k-1}\right] \vdash \tau\left\langle\mu_{0}, \cdots, \mu_{k-1}\right\rangle \mathfrak{A}$ which leads up to $\vdash \mathfrak{A}$ by assumption of induction.

Lemma 5. $\vdash \mathfrak{A}$ if and only if there is a sequence of factors $\left\{\mu_{0}, \cdots, \mu_{k}\right\}$ $(n \geqq k \geqq 1)$ such as

$$
\begin{aligned}
& {\left[\tau\left\langle\mu_{0}, \cdots, \mu_{k}\right\rangle \mathfrak{X}\right] \in\left\{\left[\mu_{1}\right], \cdots,\left[\mu_{k}\right]\right\} } \\
& \text { or } \quad\left[\mu_{1}\right], \cdots,\left[\mu_{k}\right] \vdash\left[\tau\left\langle\mu_{0}, \cdots, \mu_{k}\right\rangle \mathfrak{A}\right]
\end{aligned}
$$


where the $\mu$-condition holds.

Proof. Firstly by assuming $\vdash \mathfrak{A}$, we have $\left[\mu_{1}\right] \vdash \tau\left\langle\mu_{0}, \mu_{1}\right\rangle \mathfrak{A}$ by lemma 3 . If the inference for the step $\underline{s}) \tau\left\langle\mu_{0}, \cdots, \mu_{m-1}\right\rangle \mathfrak{A}$ is not [F], it must be [ $\boldsymbol{I}^{*}$ ] or $\left[\boldsymbol{U}^{*}\right]$ and then there are steps $\left.\underline{\boldsymbol{s}} \boldsymbol{A}\right)\left[\mu_{m}\right]$ and $\left.\underline{\boldsymbol{s}}\right) \tau\left\langle\mu_{0}, \cdots, \mu_{m}\right\rangle \mathfrak{A}$ in this proof-note, where the $\mu$-condition holds. However, $m$ can not exceed $n$ so that there is $k(k \leqq n)$ such as the inference for the step $\underline{\boldsymbol{p}}) \tau\left\langle\mu_{0}, \cdots, \mu_{k}\right\rangle \mathfrak{A}$ is $[\boldsymbol{F}]$. And therefore, $\left[\tau\left\langle\mu_{0}, \cdots, \mu_{k}\right\rangle \mathfrak{N}\right]$ belongs to the set of assumption steps of the step $\boldsymbol{p}$ or is deducible from the assumption steps of the step $\underline{\boldsymbol{p}}$.

Conversely, by assuming the condition, from the step $s)\left[\tau\left\langle\mu_{0}, \cdots, \mu_{k}\right\rangle \mathfrak{A}\right]$ we have the step $\underline{p}) \tau\left\langle\mu_{0}, \cdots, \mu_{k}\right\rangle \mathfrak{A}$ by $[\boldsymbol{F}]$, and the assumption steps of the step $\underline{\boldsymbol{s}}$ is also those of the step $\underline{\boldsymbol{p}}$ so that we have $\vdash \mathfrak{A}$ by lemma 4 .

Lemma 6. $\Gamma \vdash[\mathfrak{B}]$ if and only if there is a sequence of factor $\left\{\nu_{0}, \cdots, \nu_{m}\right\}$ such as

1) $\left[\sigma\left\langle\nu_{0}, \cdots, \nu_{m}>\mathfrak{B}\right] \in \Gamma\right.$

and 2) for any $\sigma$-formula $\nu_{j}(1 \leqq j \leqq m) \Gamma \vdash \nu_{j}$.

Proof. Firstly we assume $\Gamma \vdash[\mathfrak{B}]$, and let the final step of this proofnote which is arranged in the fundamental order of its index-words be $\boldsymbol{s}$ (i.e., $\boldsymbol{s}$ [이). We may prove the conclusion by induction referring to the step $\underline{s}$.

If the step $\underline{s}$ is the first step, the step $\underline{s}$ is the assumption step of itself and the $\sigma$-construction with kernel $\mathfrak{B}$ by the empty factor i.e., $\sigma<>\mathfrak{B}$. In this case, $m=0$ and the condition 2) is omitted.

Otherwise, we assume the assertion for any step $\underline{r}$ which takes precedence of $\boldsymbol{s}$ in the fundamental order.

The formula of the step $\underline{s}$ is clad so that the step $\underline{s}$ is either an assumption step of itself (case 1), or a step deduced from a step $\underline{\boldsymbol{u}}$ by $[\boldsymbol{U}]$ (case 2), or from steps $\underline{\boldsymbol{u}}$ and $\underline{\boldsymbol{v}}$ by [I] (case 3 ).

In the case 1 , the assertion holds evidently.

In the case 2 , the step $\underline{\boldsymbol{u}}$ is a clad formula and is a $\sigma$-construction with kernel $\mathfrak{B}$ by a $\sigma$-variable i.e., $\underline{\boldsymbol{u}})[\sigma\langle\nu\rangle \mathfrak{B}]$. And the step $\underline{\boldsymbol{u}}$ takes precedence of $\underline{s}$ in the fundamental order so that there is an assumption step $\underline{\boldsymbol{p}}$ of $\underline{\boldsymbol{u}}$ which is a $\sigma$-construction with kernel $\sigma\langle\nu\rangle \mathfrak{B}$, by our assumption of induction. The step $\underline{\boldsymbol{p}}$ is an assumption step of $\underline{\boldsymbol{s}}$, and a $\boldsymbol{\sigma}$-construction 
with kernel $\sigma\langle\nu\rangle \mathfrak{B}$ is also a $\sigma$-construction with kernel $\mathfrak{B}$. Thus the assertion holds in the step $\underline{s}$.

In the case 3, one of the steps $\underline{\boldsymbol{u}}$ and $\underline{\boldsymbol{v}}$ is a clad formula and the other is a bare formula by $[\boldsymbol{I}]$, and both of them take precedences of $\underline{s}$ in the fundamental order.

We would like to suppose the step $\underline{\boldsymbol{u}}$ is clad, then there is an assumption step of $\underline{s}$ whose formula is $\sigma$-construction with kernel $\mathfrak{B}$ as we had it in the case 1 , where $\nu$ is a $\sigma$-formula in this case. And then, the step $\underline{v}$ is the same bare formula as $\nu$ and is deduced from its assumption steps. The assumption steps of $\underline{v}$ are the assumption steps of $\underline{s}$ too. Thus the assertion also holds in this case.

Conversely, we have $\Gamma \vdash[\mathfrak{B}]$, by assuming the fact that there is a sequence of factor $\left\{\nu_{0}, \cdots, \nu_{m}\right\}$ such as the conditions 1) and 2) hold for any formula $\mathfrak{B}$, by induction as follows;

If $m=0$, the assertion is trivial.

We assume the assertion holds for any $i(i<m)$.

If $\nu_{m}$ is a free variable, we can deduce the step $\left.\boldsymbol{s}\right)\left[\sigma\left\langle\nu_{0}, \cdots, \nu_{m-1}\right\rangle \mathfrak{B}\right]$ from the step $\underline{\boldsymbol{u}})\left[\boldsymbol{\sigma}\left\langle\nu_{0}, \cdots, \nu_{m-1}, \nu_{m}\right\rangle \mathfrak{B}\right]$ of $\Gamma$ by $[\boldsymbol{U}]$.

If $\nu_{m}$ is a formula, according to the condition 2), we have a step $\left.\underline{\boldsymbol{v}}\right) \nu_{m}$ which is deduced from its assumption steps $\Gamma$, and the step $\underline{\boldsymbol{u}})\left[\sigma\left\langle\nu_{0}, \cdots\right.\right.$, $\left.\nu_{m-1}, \nu_{m}>\mathfrak{B}\right]$ of $\Gamma$ by the condition 1$)$. Then we can deduce the step $\underline{\boldsymbol{s}}$ ) $\left[\sigma\left\langle\nu_{0}, \cdots, \nu_{m-1}\right\rangle \mathfrak{B}\right]$ from the steps $\underline{\boldsymbol{u}}$ and $\underline{\boldsymbol{v}}$ by $[\boldsymbol{I}]$. In the both cases of $\nu_{m}$, the steps of $\Gamma$ are also the assumption steps of $\underline{s}$.

Thus we have the conclusion.

\section{(2. 2) Theorem.}

From the preceding Lemmas 5 and 6 we have the main theorem and its corollaries.

Theorem. Let $\mathfrak{A}$ be any LO-formula which has its regular parsing form by $\pi$-factors $\left\{\lambda_{0}, \cdots, \lambda_{n}\right\}$. In the primitive logic $[\boldsymbol{L O}]$, the provability of a given proposition $\mathfrak{A}$ is reducible to the fact that there are $\tau$-factors $\left\{\mu_{0}, \cdots, \mu_{k}\right\}(k \leqq n)$ and $\sigma$-factors $\left\{\nu_{0}, \cdots, \nu_{m}\right\}$ which satisfy the following conditions;

1) The $\tau$-kernel of $\mathfrak{A}$ by $\tau$-factors $\left\{\mu_{0}, \cdots, \mu_{k}\right\}$ which satisfies the $\mu$-condition of lemma 4 , is deducible from the assumptions $\left\{\left[\mu_{1}\right], \cdots,\left[\mu_{k}\right]\right\}$,

2) $\left\{\left[\mu_{1}\right], \cdots,\left[\mu_{k}\right]\right\}$ contains a $\sigma$-construction by $\sigma$-factors $\left\{\nu_{0}, \cdots, \nu_{m}\right\}$ with that $\tau$-kernel of $\mathfrak{A}$ by $\tau$-factors $\left\{\mu_{0}, \cdots, \mu_{k}\right\}$ as its kernel, 
3) Any $\sigma$-formula $\nu_{j}$ such as $1 \leqq j \leqq m$, is deducible from the assumptions $\left\{\left[\mu_{1}\right], \cdots,\left[\mu_{k}\right]\right\}$.

COROLlary 1. If there is no $\sigma$-construction of $\tau$-kernel of $\mathfrak{A}$ in the set of $\tau$ factors of $\mathfrak{A}, \mathfrak{A}$ is unprovable.

COROLlary 2. Without assumption, any elementary proposition is unprovable.

This theorem seems to suggest a procedure to find a proof-note of a given proposition which is provable in the primitive logic [LO ]. But we would like to mention about it in our later paper ([1]-part 3).

Finally, I would like to thank Professor Katuzi Ono for his guidance and his helpful suggestions.

\section{REFERENCES}

K. Ono, [ 1] On a practical way of describing formal deductions, Nagoya Math. J., 21 (1962), $115-121$.

[2] A certain kind of formal theories, Nagoya Math. J., 25 (1965), 59-86.

[ 3 ] On universal character of the primitive logic, Nagoya Math. J., 27 (1966), 331-353.

[4] Reduction of logics to the primitive logic, J. Math. Soc. Jap., Vol. 19, No. 3 (1967), 384398.

[5] A study on formal deductions in the primitive logic, Nagoya Math. J., 31 (1968), 1-14.

J. Ito, [1] Studies on mechanical deductions part 1, Jour. Toyota Technical College, Vol. 1 (1968), 41-49.

Toyota Technical College 